\title{
Passive Event-Based Extrapolation for Lossy Haptic Data Compression in Bilateral Presence Systems
}

\author{
Philipp Kremer and Martin Kuschel and Carsten Preusche and Martin Buss, and Gerd Hirzinger
}

\begin{abstract}
A new lossy compression method is proposed for haptic (force, velocity) data as exchanged in bilateral telepresence systems. The method is based on the passive extrapolative compression strategy proposed in [1]. The innovation is that the extrapolations do not have a stiff horizon, but are triggered by considerable changes (events) in the target environment. This enables longer average extrapolation horizons and thus, higher compression. Experiments are conducted using two DLR Light Weight Robots. The results indicate that the method outperforms older implementations.
\end{abstract}

\section{INTRODUCTION}

Presence systems allow humans to operate in two kinds of target environments: Virtual reality systems allow humans to immerse in an artificially generated environment. Telepresence systems allow humans to immerse in a real, but inaccessible environment. The inaccessibility can be due to distance, scaling or living conditions. A bilateral presence system consists of a human operator who commands an avatar/teleoperator (TO) in the virtual/remote environment. A multimodal human system interface (HSI) is used for the operator to command the TO and, concurrently, to display the target environment. Signals are exchanged over a communication channel (COM). See Fig. 1 for an illustration.

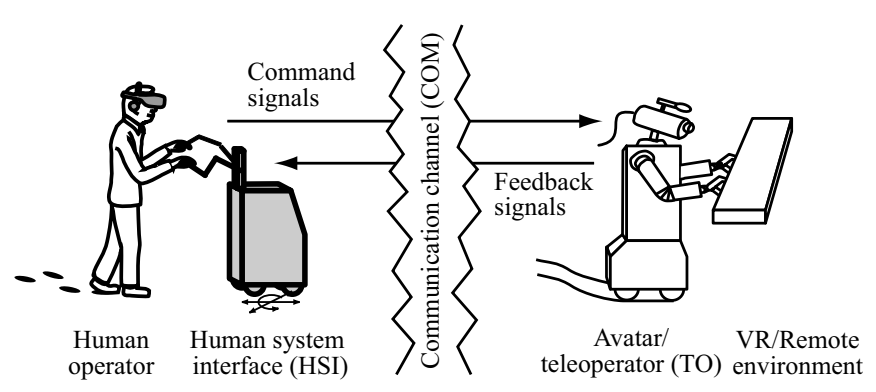

Fig. 1. Multimodal bilateral presence system: A virtual or remote environment is mediated via technological equipment.

Presence systems that enable realistic, immersive experiences are usually equipped with highly dexterous facilities to perform and display kinesthetic-haptic stimuli. In such a system HSI an TO are equipped with haptic devices that have several degrees of freedom $(>20)$ each using highly

P. Kremer, C. Preusche, and G. Hirzinger are with the Institute of Robotics and Mechatronics, German Aerospace Center (DLR), Wessling, Germany philipp.kremerdalr.de

M. Kuschel and M. Buss are with the Institute of Automatic Control Engineering, Technische Universität München, Germany; M. Kuschel also is employed at KUKA Roboter GmbH, Augsburg, Germany martin.kuscheldtum.de accurate sensors ( $>14$ bit) and actuators controlled at high sample rates $(>1 \mathrm{kHz})$. Hence, for the bilateral information exchange between HSI and TO, a large amount of data has to be exchanged over the COM. Since bandwidth is often limited (e.g. existing bus systems) or costly (e.g. space communications) the need for effective compression algorithms for haptic data is salient.

In bilateral, kinesthetic-haptic (by now 'haptic') telepresence systems, force and position-based signals are exchanged between operator and target environment. Thereby, a control loop is closed between HSI and TO. Main objectives in the control system design are stability and transparency. Ideal transparency means, that the operator does not perceive the presence-mediating technology (HSI, COM, TO) when experiencing the target environment.

Compression algorithms reduce the amount of data to be stored, processed, or transmitted. The design of compression algorithms for haptic data raises three main issues. Firstly, high compression ratios are desired. Secondly, the compression should not be observable by the human operator, i.e. it should be transparent. Thirdly, the compression should not destabilize the telepresence system. While the first two issues are typical for all compression problems, the third issue does only arise in compression problems for haptic data in bilateral systems, such as telepresence- and VR-systems. Several compression methods for haptic data can be reviewed in the literature. The method proposed in [2] results in a trade-off between compression efficiency and delay required for compression. Differential pulse code modulation (DPCM) together with a fixed rate quantization has been proposed in [3]. Adaptive DPCM together with Huffman coding has been considered in [4]. General considerations about haptic lossy compression methods and perceptual performance have been presented in [5]. The first approach that assures the stability of the overall system including delayed communication) is deadband control introduced in [6], [7]. The method can achieve large average compression ratios. However, an important drawback of the algorithm is its inability to maintain an upper bandwidth limitation. If force or velocity signals are subject to fast changes the compression savings can decrease to zero. The first fix-data-rate compression methods for haptic data with stabilization for delayed communication were proposed in [1]. The compression methods are based on interpolative and extrapolative compression strategies. In this article we propose a new compression method based on the passive extrapolative strategy that we published in [1]. The method features an event-based extrapolation. Thereby, a new extrapolation can be started if a considerable change 
(event) in the environment occurs. Simulations and experiments indicate that the method outperforms the extrapolative downsampling method, proposed in [1], while maintaining an upper bandwidth limit.

The remainder is organized as follows: In Section II background information is given about compression, stability, transparency, and extrapolative compression. In Section III the new algorithms are explained. Simulations are provided in Section IV. Experimental results are shown in Section V. A conclusion is drawn in Section VI.

\section{Theoretical BACKground}

\section{A. Data Compression Ratio}

The data compression ratio is defined by the ratio of the original data rate [bit/s] to the reduced data rate yielding

$$
\mathrm{CR}=\frac{\text { Uncompressed Data }}{\text { Compressed Data }} \text {. }
$$

High compression ratios are desirable $\mathrm{CR}>>1$.

\section{B. Transparency}

A presence system is called transparent if the human operator cannot distinguish between the target and the mediated environment. For haptic presence systems objective criteria like the synchronicity of positions and forces on operator and teleoperator side [8]

$$
x_{t}=x_{o} \quad \text { and } \quad f_{t}=f_{o}^{e},
$$

give advice whether a certain system is transparent or not. See Fig. 2 for an illustration of the impedance structure of a telepresence system ( $x$ denotes position, $v$ velocity, and $f$ force). Residuals are caused by all involved subsystems. In data compression the residuals are called artifacts. Hence, a lossy data compression (LDC) algorithm is called transparent if it does not cause any artifacts. Artifacts have two origins. Firstly, they can be caused by the loss of information leading to reconstruction failures (approximation artifacts). Secondly, artifacts can be caused by the delay introduced by the compression algorithm (phase artifacts).

\section{Passive Haptic Compression}

In contrast to audio and video compression, haptic compression algorithms are applied within an energy-exchanging closed control loop between operator and environment. Velocity and force signals are exchanged. The dynamics of the compression algorithm within this control loop can render the presence system instable. To assure stability the passivity paradigm can be deployed. The argumentation is that a system comprised of passive subsystems remains passive if its subsystems are connected in parallel or feedback structure. The passive subsystems of a bilateral presence system are illustrated in the upper diagram of Fig. 2.

A reachable dynamic system in velocity-force architecture with zero initially stored energy is passive if

$$
\int_{0}^{t} P_{\text {in }} \mathrm{d} \tau \geq 0, \quad \forall t \geq 0,
$$

where $P_{i n}$ is the power input of the system. For the COM of a presence system in velocity-force architecture, the input power is defined as the scalar product of force and velocity

$$
P_{\text {in }}=v_{o} f_{t}^{c}-v_{o}^{c} f_{t}
$$

where $v_{o}$ is the velocity commanded by the operator, $v_{o}^{c}$ is the commanded velocity on teleoperator side, $f_{t}$ is the force reflected by the teleoperator, and $f_{t}^{c}$ is the reflected force on operator side. The power entering the system is counted positive and the power leaving the system is counted negative.

In many applications the COM of a presence system, especially in telepresence architecture, is afflicted with communication delay (due to delay in the communication networks like the Internet, etc.). Delays are active elements. Therefore, passivity measures for the COM are mandatory. Usually, the Scattering transformation is applied to passivate the COM in presence of constant communication delay (varying delays are buffered to yield a constant delay) mapping power variables (velocity, force) into wave variables

$$
\begin{gathered}
g_{l}=\frac{b v_{o}+f_{t}^{c}}{\sqrt{2 b}}, \quad h_{l}=\frac{b v_{o}-f_{t}^{c}}{\sqrt{2 b}}, \\
g_{r}=\frac{b v_{t}^{c}+f_{t}^{e}}{\sqrt{2 b}}, \quad h_{r}=\frac{b v_{t}^{c}-f_{t}^{e}}{\sqrt{2 b}} .
\end{gathered}
$$

Thereby, $g_{l}, h_{r} \in \mathbb{R}$ denote the incident wave and $g_{r}, h_{l} \in \mathbb{R}$ denote the reflected wave (also called wave reflections). The indices denote the waves on the right or on the left side. The parameter $b$ (wave impedance) is a positive constant that can be chosen arbitrarily. The transformation is bijective, i.e. unique and invertible. Hence, no information is lost or gained by encoding power variables into wave variables or wave variables into power variables. The passivated COM is depicted in the lower diagram of Fig. 2.

Applying the Scattering transformation Eq. (5) to the power input of a telepresence system Eq. (4) yields the power input expressed in Scattering variables

$$
P_{i n}=\frac{1}{2}\left(g_{l}^{2}-g_{r}^{2}+h_{r}^{2}-h_{l}^{2}\right)
$$

where the index indicates the wave variables on the right and on the left hand side. Latter equation can be divided into a passivity condition for systems in the command path of the Scattering domain

$$
\int_{0}^{t} g_{l}^{2}(\tau) \mathrm{d} \tau \geq \int_{0}^{t} g_{r}^{2}(\tau) \mathrm{d} \tau
$$

and a passivity condition for systems in the reflection path of the Scattering domain

$$
\int_{0}^{t} h_{l}^{2}(\tau) \mathrm{d} \tau \leq \int_{0}^{t} h_{r}^{2}(\tau) \mathrm{d} \tau
$$

This equation illustrates that waves carry their own power (unit of measurement $\sqrt{W}$ ). Hence, systems in wave domain remain passive if the output wave does not carry more energy than the input wave.

Since the passivation of the COM is mandatory in haptic telepresence, LDC-methods should also retain the passivity 


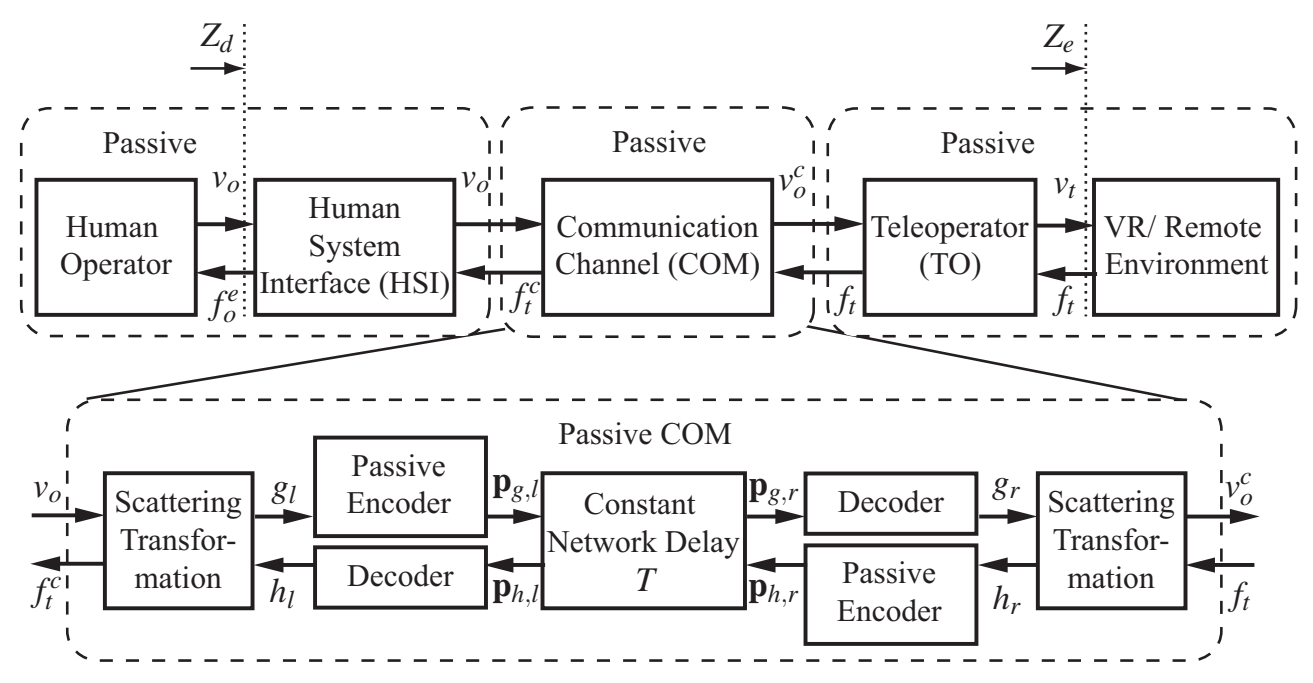

Fig. 2. Presence system in two-port, velocity-force architecture: (Upper diagram) If the subsystems [operator-HSI], COM, and [TO- environment] are passive and connected in parallel or feedback connection, the overall system is passive, i.e. stable, as well. If serial connections occur the system is not passive but remains stable. Sensor dynamics in HSI and TO are omited. (Lower diagram) Structure of the COM for passive compression: Encoder and decoder are applied in Scattering domain, which assures passivity for arbitrary constant delays. Furthermore, encoder and decoder have to be passive themselves.

of the COM. Hence, the compression algorithm has to be passive in Scattering domain.

Additional measures to passivate delay caused by the LDCalgorithm are not necessary since the Scattering transformation already passivates constant communication delays, which includes possible delay introduced by the compression. The passivation causes phase artifacts. According to [9], [10] these phase artifacts also depend on the dynamics of the target environment.

The amplitude change caused by compressing the signal is not passive and not passivated by the Scattering transformation. Hence, it has to be passivated by additional measures to obey the passivity conditions in wave domain Eqs. (7), (8).

\section{Extrapolative Compression Strategy}

The extrapolative strategy as proposed in [1] estimates future samples to a certain extent, called extrapolation horizon $T_{E H}$. The encoder works as follows: $k_{E H}$ samples are estimated and a signal is constructed based on certain assumptions resulting in the parameter vector $\mathbf{p}$ transmitted over the network and reconstructed by the decoder.

Every $k_{E H}$ samples an extrapolation of the next $k_{E H}$ samples is performed. The duration of the extrapolation horizon amounts to

$$
T_{E H}=\frac{k_{E H}}{f_{s}} .
$$

The compression principle is depicted in Fig. 3.

The amplitude changes introduced by the extrapolation procedure depends on the energy difference between original signal and estimated signal. According to conditions Eqs. (7), (8) passivity of the amplitude change can be assured by forcing the extrapolated wave to contain equal or less energy than the difference between the original wave and the preceding extrapolations starting from the beginning. Hence, the passivity criterion for the extrapolative compression strategy is

$$
\int_{0}^{t_{j}} g_{l}^{2} \mathrm{~d} t-\int_{0}^{t_{j}+T} g_{r}^{2} \mathrm{~d} t \geq \int_{t_{j}+T}^{t_{j}+T_{E H}+T} g_{r}^{2} \mathrm{~d} t,
$$

with $t_{j}$ representing the time when a new extrapolation is performed and $T_{E H}$ as the length of the extrapolation horizon. The compression ratio is

$$
\mathrm{CR}=\frac{k_{E H}}{\operatorname{dim}(\mathbf{p})} .
$$

Transparency is influenced only by approximation artifacts resulting from the extrapolation. The advantages of the extrapolative compression are:

1) A constant, freely adjustable data rate. Hence, any communication bandwidth limits can be satisfied.

2) No strategy-inherent delay. Hence, no phase artifacts will deteriorate transparency.

3) Arbitrary algorithms are possible, as long as condition Eq. (10) is satisfied.

\section{E. Extrapolative Downsampling}

An implementation using the extrapolative compression strategy proposed in [1] is passive extrapolative downsampling (eDS). The main idea is to extrapolate the future signal by a single value within a stiff extrapolation horizon $T_{E H}$. The extrapolation is based on a hold last sample (HLS) procedure that was modified to satisfy the passivity criterion for the extrapolative compression strategy Eq. (10),

\section{PASSIVE EVENT-BASED EXTRAPOLATIVE COMPRESSION}

The quality of extrapolations is limited by constraint information about the dynamics of the target environment, i.e. by constraint information about the future. The longer the algorithm extrapolates the future, the less accurate it will be, if the environment changes. Hence, a transparent 

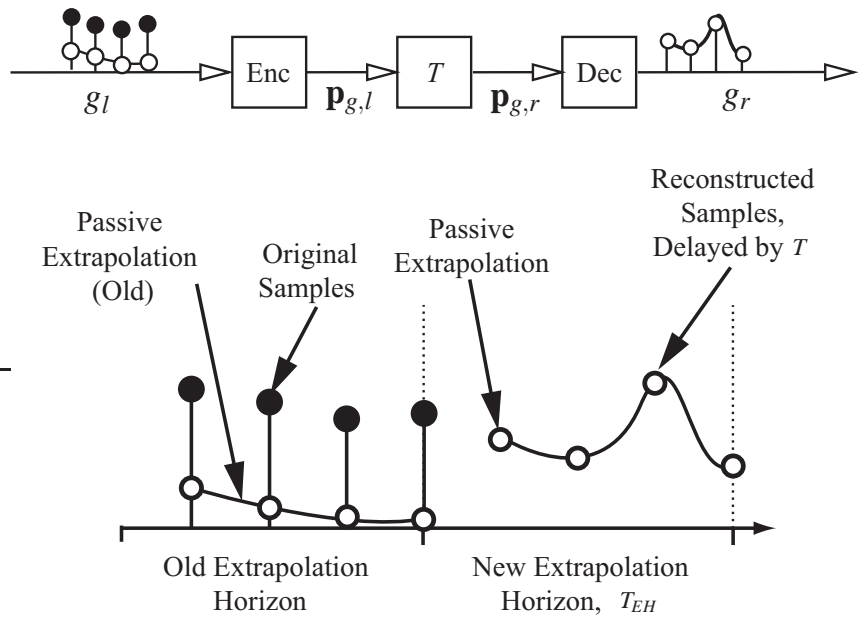

Fig. 3. Principle of the extrapolative compression strategy as proposed in [1] : The signal within the extrapolation horizon is anticipated using the residual energy difference between precedent extrapolations and its real correlates.

parametrization results in a rather short extrapolation horizon to account for sudden changes in the environment. But if the environment changes slowly, the horizon could be larger resulting in higher compression ratios. Algorithms with stiff extrapolation horizons, such as eDS, lack an environmentdepending extrapolation horizon and are therefore, inefficient if the environment changes slowly, e.g. in free space.

To account for considerable changes in the environment, additional information about the target environment can be fed back modulating the length of the extrapolation horizon. This is the main idea of the passive event-based extrapolation.

\section{A. Event-Based Extrapolative Compression (eEB)}

The algorithm is based on a stiff extrapolation horizon, which can be changed if an event occurs. Thereby, the most recent value is used as extrapolation value, if it fullfills the passivity conditon Eq. (10). Otherwise a value is calculated to fullfill this equation. The reconstructed value is choosen

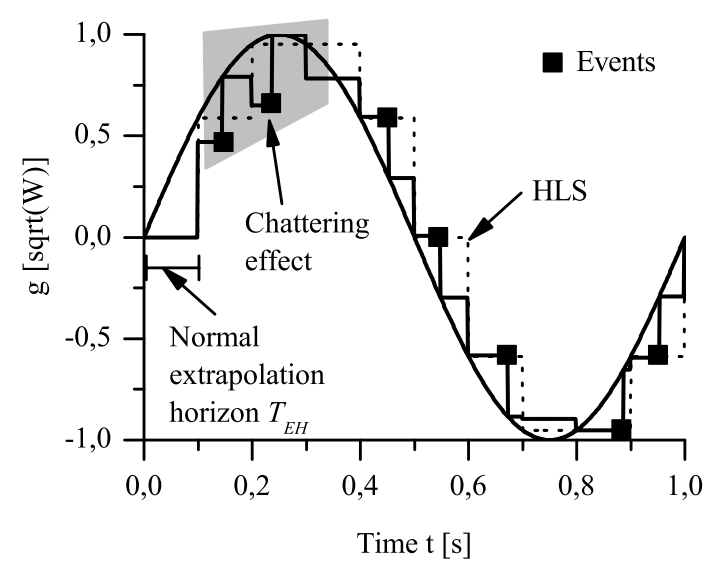

Fig. 4. Operation mode of eEB $\left(T_{E H}=0.1 \mathrm{~s}, f_{s}=1 \mathrm{kHz}\right)$ : The algorithm generates a chattering effect if an event-based extrapolation leaves too little energy for a proper succeeding extrapolation. (Network delay is $T=0$.) to be zero, if no packet arrived at the decoder

$$
g_{r}(t)=0, \forall t<T \text {. }
$$

For the sample instants at which an extrapolation takes place, the reconstructed signal is calculated according to

$$
\begin{gathered}
g_{r}(t)= \begin{cases}g_{l}(t-T) & \text { if (10) holds, } \\
g_{l} & \text { and such that (10) holds, }\end{cases} \\
\text { if } t>T \vee t-T=k T_{\mathrm{EH}}, \quad k \in N .
\end{gathered}
$$

To account for considerable changes in the environment an additional packet, which contains information representing the actual environment, can be sent within every extrapolation horizon. This packet is called event. Events are defined by the following rules:

- The absolute difference between the last transmitted sample and the most recent original sample must exceed a positive constant threshold $\eta$

$$
\left|g_{l}\left(t_{j}\right)-g_{l}(t)\right|>\eta_{g}, \quad \eta_{g}>0 .
$$

- Only a single event is allowed per extrapolation horizon.

- The extrapolation is updated by the new value, which must fullfill the passivity condition Eq. (10).

If an event has taken place, the updated extrapolation is valid until the stiff extrapolation horizon ends. These requirements are summarized in the following equation:

$$
g_{r}(t)= \begin{cases}g_{l}(t-T) & \text { if (10) holds } \vee(14), \\ g_{r}\left(t-t_{s}\right) & \text { otherwise }\end{cases}
$$

if neither Eq. (12) nor Eq. (13) are valid.

Where $t_{s}$ denotes the sampling time. The basic principle is illustrated in Fig. 4. As can be seen, the algorithm can generate a chattering effect. A chattering effect occurs if the energy of the updated extrapolation leaves too little energy for a properly updated extrapolation.

Event-based extrapolation allows for a lower limit of the compression ratio. Therefore, it is assumed that the COM can transmit packets at the sampling frequency of the presence system. This compression algorithm differs from exclusive stiff extrapolation, as there is not a single possible compression ratio, but a certain upper and lower limit. The limits of the compression ratio are $\mathrm{CR}_{\mathrm{eEB}}=2 \mathrm{CR}_{\text {stiff }}$ and $\mathrm{CR}_{\mathrm{eDS}}=$ $\mathrm{CR}_{\text {stiff, }}$, if the event packets are not discernable from normal packets $\left(\mathrm{CR}_{\text {stiff }}\right.$ denotes compression algorithms with stiff extrapolation horizon and single parameter extrapolation). In the worst case scenario an event is triggerd within every extrapolation horizon. Whereas, in the case no events are triggered the compression ratio equals the stiff compression ratio.

For communcation environments with specially tailored protocolls, in which compression schemes with fixed upper data rates are especially sensible, like realtime space communciation, a much better worst case compression rate is to be expected. Event packets can by their nature be drastically reduced in terms of quantization, as they only need to feature a limited range and a low resolution. By reducing their quantization by half, limiting their range from threshold to 
half a full change, both with respect to normal packets, and transmitting only the difference, the compression ratio should increase to an overhead of a fourth,

$$
\mathrm{CR}_{\mathrm{eEB}}=1.25 \mathrm{CR}_{\mathrm{eDS}} \text {. }
$$

\section{B. Extended eEB (eEBext)}

To avoid the chattering effect of the eEB, the requirements for an event are extended. In comparison to eEB, a certain amount of energy must be left available. This amount is inversely proportional to the remaining extrapolation horizon, according to

$$
\frac{\sqrt{\int_{0}^{t_{j}} g_{l}^{2} \mathrm{~d} t-\int_{0}^{t_{j}+T} g_{r}^{2} \mathrm{~d} t}}{2 T_{\mathrm{EH}}-\left(t-t_{j}\right)} \geq g_{l}^{2}(t)\left(T_{\mathrm{EH}}-t+t_{j}\right)
$$

The eEBext is illustrated in Fig. 5. The chattering behaviour is avoided since no events are triggered, if too less energy is available to update the remaining extrapolation horizon with the new value.

\section{SIMULATIONS}

\section{A. Method}

A simulation was conducted to unveil the basic performance of compression algorithms eEB and eEBext in comparison to eDS.

The operator was modeled as a velocity source using a sum of a step at $37.627 \mathrm{~s}$ of size -1 and a chirp signal with frequencies from $.001 \mathrm{~Hz}$ to $1 \mathrm{~Hz}$ an amplitude of 1 , starting with the simulation and reaching the final frequency at half the simulation time. Simulation parameters are $T_{\mathrm{sim}}=100 \mathrm{~s}$, and $f_{s}=1 \mathrm{kHz}$. The force-controlled HSI was modeled by a transfer function of $G_{h i s}=\frac{1}{s+1}$ ( $s$ denotes the complex frequency). The TO and environment were modeled by a transfer function $Z_{t o}=\frac{s}{s+.5}$ with an external force step of size -1 at $11.345 \mathrm{~s}$. The compression algorithms were parametrized to obey the minimal compression ratio 1 : 10. The extrapolation horizon of eEB and eEBext was 20 samples. The extrapolation horizon of eDS was 10 samples. The threshold to trigger the events for eEB and eEBext was

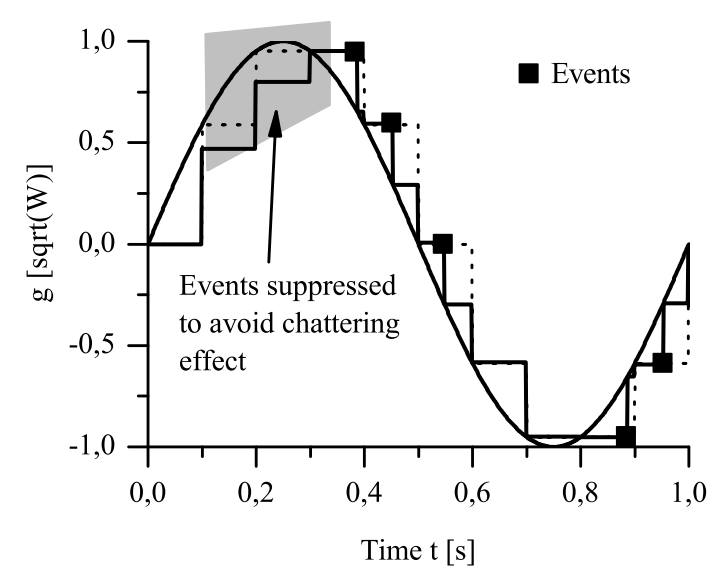

Fig. 5. Operation mode of eEBext $\left(T_{E H}=0.1 \mathrm{~s}, f_{s}=1 \mathrm{kHz}\right)$ : Chattering effects are avoided. (Network delay is $T=0$.)

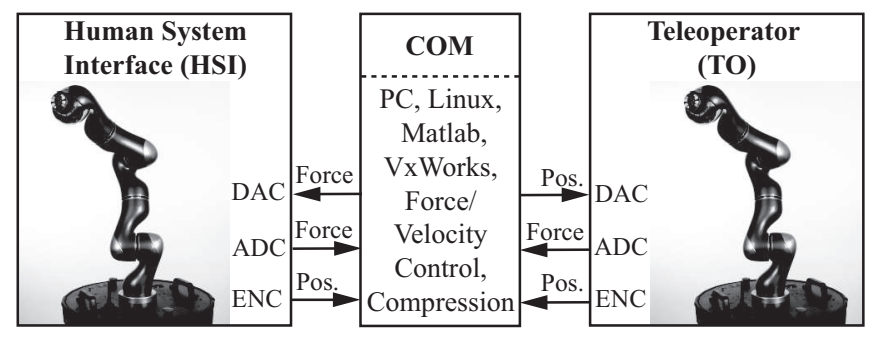

Fig. 6. Presence system: The telepresence system consisted of a haptic HSI, a haptic TO, and a real-time processing unit that emulated the COM. For the HSI and the TO high fidelity DLR Light Weight Robots (third generation) were used. Positions and forces were measured and exchanged. The COM was passivated for constant delays.

chosen asymmetric, since the incident wave in our simulation had a greater average amplitude than the reflected wave. Therefore, the threshold for events on the incident wave was $\eta_{g}=0.5$, for events on the reflected wave the threshold was $\eta_{h}=0.3$. The criterion to evaluate transparency was based on the criterion Eq. (2). Hence, the transparency criterion was comprised of the normalized position and force errors

$$
T_{v}=\frac{x_{o}-x_{t}}{T_{\operatorname{sim}} f_{s}}, \quad T_{f}=\frac{f_{t}-f_{t}^{c}}{T_{\operatorname{sim}} f_{s}} .
$$

\section{B. Results and Discussion}

The results of the transparency analysis are summarized in Table I. The position error of eEB is smaller than eDS, even though the extrapolation horizon was chosen to be twice as high. The position error of eEBext was greater than the position error using eDS. The force errors are smaller in both new algorithms eEB, eEBext compared to eDS. By choosing different thresholds that govern the generation of events in the reflected and in the incident wave, the results could vary, but the general tendency is obvious: The new algorithms provide higher performance compared to extrapolative downsampling (eDS).

\section{EXPERIMENTS}

\section{A. Method}

1) Presence System: A telepresence system was used in velocity-force architecture (Fig. 2) that provided haptic command and feedback signals at high accuracy. The system consisted of two DLR Light Weight Robots of the third generation and is illustrated in Fig. 6. The DLR Light Weight Robot (LWR) is a light-weight, flexible, revolute joint robot, which by its overall sensoric equipment is especially dedicated to work in human-robot interaction scenarios. A detailed description of the robot is provided in [11]. The COM was emulated by a PC that also hosted the realtime processing environment (VxWorks) to perform the force and velocity control as well as the compression algorithms.

TABLE I

SimUALTION RESULTS

\begin{tabular}{|c||c|c|c|c|}
\hline Criterion & uncompressed & eDS & eEB & eEBext \\
\hline \hline$T_{v}$ & $10^{-3}$ & 0.33 & 0.01 & 0.81 \\
\hline$T_{f}$ & 0.02 & 0.11 & 0.04 & 0.04 \\
\hline
\end{tabular}


-.-- $f_{t}$ (reflected force)

$-\mathrm{f}_{\mathrm{t}}^{\mathrm{c}}$ (displayed force)

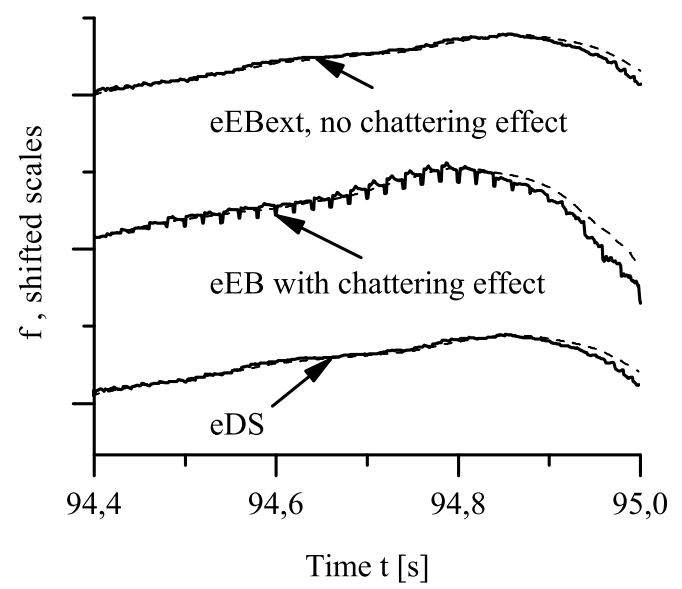

Fig. 7. Experiment: Stable telepresence was performed with the new compression algorithms applied. The chattering effect generated by eEB became salient in the force data. The extension of the algorithm eEBext successfully suppressed this effect and approximation artifacts were similar to eDS.

Communication time was set to one sample time step, in order to start with a high fidelity system.

2) Participants, Stimuli, and Procedure: The experiment was conducted with a single participant operating the presence system in three different environments. The stiff environment had a compliance of $2 \mathrm{~mm} / \mathrm{N}$, the soft environment had a compliance of $14 \mathrm{~mm} / \mathrm{N}$, the third environment was free space having infinite compliance. The operator arbitrarily commanded the teleoperator to the different environments. The experiment was designed to analyze the two algorithms (eEB, eEBext) objectively, i.e. for each algorithm a certain trial was conducted and objective velocity and force information was recorded.

\section{B. Results and Discussion}

Stable telepresence could be performed with our compression algorithms applied. A cutout of the experimental trajectory showing force data is depicted in Fig. 7. The chattering-effect generated by eEB is reduced using the extended event-based extrapolation eEBext. Descriptively, nearly no difference between the old eDS and eEBext could be registered, despite the overall increase of compression ratio. Since the operator's command signal was different in each trial, an objective comparison between the algorithms was not possible with this experiment, but the main features could be illustrated.

\section{CONCLUSIONS AND FUTURE WORKS}

Based on the passive, extrapolative compression strategy proposed in [1], we developed new algorithms that perform passive, extrapolative compression with an environmentdepending extrapolation horizon. The first algorithm (eEB) starts a new extrapolation each time an event is triggered, thereby, obeying the passivity condition Eq. (10). Since eEB generated a chattering effect, we developed a version (eEBext) that suppressed event-triggered extrapolations when chattering could occur.

Using event-based (eEB, eEBext) instead of stiff (eDS) extrapolation horizons, a more efficient coding is possible, since slow changing environments can be encoded with a higher compression ratio. The simulations showed that both algorithms outperform a compression algorithm with stiff extrapolation horizon (eDS). The new algorithms enable constant, freely adjustable upper bandwidth limitations, while reducing the mean used bandwidth. For a final evaluation of the proposed algorithms a psychophysical study is necessary to identify the perceptual transparent compression ratio and a useful values for considerable changes in the environment.

\section{ACKNOWLEDGMENTS}

This research was partly funded by the German National Science Foundation (DFG) within the Collaborative Research Center on "High-Fidelity Telepresence and Teleaction" (SFB 453).

\section{REFERENCES}

[1] M. Kuschel, P. Kremer, S. Hirche, and M. Buss, "Lossy data reduction methods in haptic telepresence systems," in IEEE Proceedings of the International Conference on Robotics and Automation, Orlando, USA, 2006.

[2] C. Shahabi, A. Ortega, and M. R. Kolahdouzan, "Comparison of Different Haptic Compression Techniques," in Proceedings of the International Conference on Multimedia and Expo (ICME), Lausanne, Switzerland, 2002.

[3] C. W. Borst, "Predictive Coding for Efficient Host-Device Communication in a Pneumatic Force-Feedback Display," in Proceedings of the First Joint Eurohaptics Conference and Symposium on Haptic Interfaces for Virtual Environment and Teleoperator Systems, Pisa, Italy, 2005.

[4] A. Ortega and Y. Liu, Touch in Virtual Environments: Haptics and the Design of Interactive Systems, M. McLaughlin and J. Hespanha, Eds. Prentice Hall, 2002.

[5] M. H. Zadeh, D. Wang, and E. Kubica, "Perception-based lossy haptic compression considerations for velocity-based interactions," Multimedia Systems, vol. 13, pp. 275-282, 2008.

[6] S. Hirche and M. Buss, "Transparent data reduction in networked telepresence and teleaction systems, part I: Communication without time delay," PRESENCE: Teleoperators and Virtual Environments, vol. 16, no. 5, 2007.

[7] S. Hirche, P. Hinterseer, E. Steinbach, and M. Buss, "Transparent data reduction in networked telepresence and teleaction systems, part II: Time-delayed communication," PRESENCE: Teleoperators and Virtual Environments, vol. 16, no. 5, 2007.

[8] Y. Yokokohji, "Bilateral control of master-slave manipulators for ideal kinesthetic coupling - formulation and experiment," IEEE Transactions on Robotics and Automation, vol. 10, pp. 605-620, 1994.

[9] S. Hirche, "Haptic telepresence in packet switched communication networks," Ph.D. dissertation, Technische Universität München, 2005.

[10] M. Kuschel and M. Buss, "Impedance-based performance analysis of haptic telepresence systems," in Proceeding of the 2nd International Workshop Human Centered Robotics, Munich, Germany, 2006.

[11] G. Hirzinger, N. Sporer, A. Albu-Schäffer, M. Hähnle, R. Krenn, A. Pascucci, and M. Schedl, "Dlr's torque-controlled light weight robot iii - are we reaching the technological limits now," in Proceedings of the IEEE Int. Conference Robotics Automation, 2002, pp. 1710-1716. 